\title{
GLOBALIZING KNOWLEDGE: HOW TECHNOLOGICAL OPENNESS AFFECTS OUTPUT, SPATIAL INEQUALITY, AND WELFARE LEVELS*
}

\author{
Giulio Bottazzi \\ Istituto di Economia, Scuola Superiore Sant'Anna, Piazza Martiri della Libertà, 33, 56127 Pisa, \\ Italy; and, BRICK, Collegio Carlo Alberto, Via Real Collegio, 30, 10024, Moncalieri, Torino, Italy. \\ E-mail: g.bottazzi@sssup.it
}

\section{Pietro Dindo}

Dipartimento di Economia e Management, Università di Pisa, Via C. Ridolfi, 10, 56127, Pisa, Italy. E-mail:p.dindo@ec.unipi.it

\begin{abstract}
Using an analytically solvable model, we study how the spatial distribution of economic activities and the ensuing welfare levels are affected by pecuniary externalities, depending on transportation costs, and localized technological externalities, due to the cost saving effect of intra- and interregional knowledge spillovers. Under the assumption of capital mobility and labor immobility, we show that increasing interregional knowledge spillovers, i.e., promoting technological openness, favors a smoother transition between different levels of firms concentration, makes trade globalization less likely to generate catastrophic and irreversible agglomeration, and ultimately leads to a less uneven distribution of welfare.
\end{abstract}

\section{INTRODUCTION}

In this paper we analyze how and to what extent a variation in the degree of social and technological openness, and its interaction with trade globalization, affects the geographical distribution of firms and wealth. The skewed nature of the distribution of economic activities found in both developed and developing countries, at any scale, from cities to regions, can be the result of both market-mediated interactions, such as labor pooling or intermediate goods availability, and nonpecuniary differences across geographical locations. Indeed, beside the effect of trade openness in final markets and increased mobility in factors of production, like labor and capital, economic agglomeration is plausibly enhanced by the institutional framework, the availability of public infrastructures, higher levels of human and social capital, and the local and tacit nature of technical knowledge. In fact, the abundant presence of agglomerated production clusters away from big cities and main transport systems suggests that forces other than transportation costs, advantages due to larger local demand, or deeper factor markets, are at work. These forces are not exclusively acting in high-tech sectors, like semiconductors or ICT services, but are rather pervasive in the entire economy. Analyzing the Italian manufacturing industry, Bottazzi et al. (2008) and Bottazzi and Gragnolati (2012) find that sectors like

\footnotetext{
*We are grateful to Giovanni Dosi, Francesco Lissoni, Steven Brakman, and two anonymous referees for many useful suggestions. We also thank the participants of the DIMETIC Summer School "Geography of Innovation and Growth," Pecs, Hungary; of the First DIME Scientific Conference, Strasbourg and of the Final DIME Scientific Conference, Maastricht. The research that has led to this work has been supported by the EU FP6 STREP Project CO3 "Common Complex Collective Phenomena in Statistical Mechanics, Society, Economics, and Biology," by the European Union NoE DIME, and by MIUR, PROT. 2007HA3S72003, PRIN 2007. All usual disclaimers apply. Previous versions of this work have circulated under the title "Localized technological externalities and the geographical distribution of firms."

Received: March 2012; revised: January 2013; accepted: January 2013.
} 
food products, leather products, or basic metal workings are highly agglomerated, and their agglomeration cannot be explained by the presence of transport infrastructures or localized demand. This is not a peculiar aspect of Italian manufacturing, as similar results have been found for the U.S. (Ellison and Glaeser, 1997), France (Maurel and Sedillot, 1999), Germany (Brenner, 2006), and the U.K. (Devereux, Griffith, and Simpson, 2004).

If localized nonpecuniary advantages are important in describing the observed outcome of firms locational choice, at least as much as pecuniary market-mediated interactions, it becomes relevant to investigate which aggregate effects can be observed when the institutional, cultural, and social barriers that make these advantages local are, at least partially, abated. Among the nonpecuniary factors that presumably provide local advantages in production, a particular attention has been devoted to the possible presence of technological externalities (Marshall, 1920) induced by localized knowledge spillovers (LKS). They allow colocated companies to share part of their knowledge sources, reduce innovation costs, and gain competitive advantages with respect to firms located elsewhere. The mechanisms behind the local accrual of economic exploitable knowledge are potentially many and the empirical literature seems to lack an agreement on the general functioning of LKS. Typical proxies of corporate effort, like R\&D expenditures, or of codified output, like patents, are likely to describe only part of the story. Moreover, some issues have been raised about the actual effectiveness of the econometric models and tools adopted in their measurements, see, e.g., Breschi and Lissoni (2001a, 2001b). Whatever the specific channel behind the creation of technological advantages, it is commonly accepted that factors external to market interactions, like local institution, social convention, and legal right, contribute to it (see for instance the discussion in Dosi, Pavitt, and Soete, 1990, Chapter 8; or Audretsch and Feldman, 2004, Section 5). In this respect, the institutional proximity of different social communities matters in defining the range at which knowledge could plausibly spill. In their re-visitation of the notion of "localized learning," Malmberg and Maskell (2006) notice how the formation of international regulating and supervising authorities, the development of common commercial laws, the internationalization of the capital market, and the increased mobility of ideas are likely to alter the geographical reach of knowledge spillovers.

We address the question of the effect of technological openness on trade and welfare inside the domain of new economic geography (NEG). Since Krugman (1991b) this literature has mostly dealt with the effect of pecuniary externalities on the spatial distribution of economic activities. We advance an analytically tractable general equilibrium model that explicitly accounts for the presence of nonpecuniary technological externalities via localized knowledge spillovers. Concerning the pecuniary part of the model, we share similar assumptions with the so called "footloose capital" models (see Baldwin et al., 2003, for a review): a perfectly competitive traditional sector producing a homogeneous good using labor under constant returns to scale, together with a modern sector producing several distinct goods using fixed capital and labor under increasing returns to scale and monopolistic competition. Households preferences for modern goods are characterized by constant elasticity of substitution (CES), so that variety in the modern sector is rewarded. Following Forslid and Ottaviano (2003), we obtain analytical tractability through partial factor immobility. In particular, we impose labor immobility and assume that households are both local workers and global investors, as in Martin and Rogers (1995). In this way, the mobile factor is represented by the capital, whose rent is payed to households/shareholders and consumed in the location where they reside. We believe that this assumption well represents today increased capital mobility, specially in geographical area like the European Union, in which relative regional homogeneity leads to flows of capital which are hardly matched by flows of any other productive factor. 
The main difference between our model and the incumbent literature concerns the way in which we model localized knowledge spillovers. Despite the original lack of interest, ${ }^{1}$ recent theoretical contributions to NEG have extended the investigation by including nonpecuniary external economies. In particular, a number of works have adopted the type of externality introduced in growth models by Grossman and Helpman (1991), postulating a R\&D sector with increasing returns to scale that contributes to economic growth via a constant creation of new modern goods. Martin and Ottaviano (1999) and Baldwin, Martin, and Ottaviano (2001) include this type of externality in a footloose capital model. The results are extensively discussed in Baldwin et al. (2003), Chapter 7, where the models are referred to as global spillover (GS) and local spillover (LS). Davis (2009) extends this framework by including occupational choice between skilled and unskilled labor. Baldwin and Forslid (2000) consider the same type of technological externality, but this time with mobile workers and immobile capital. In all these models knowledge accumulation and geography are linked by the introduction of an interregional spillover parameter, a sort of "technological openness," which measures to what extent nonpecuniary advantages in the R\&D sector are location specific or can be shared across regions.

We take a different approach. We model nonpecuniary technical advantages as having a direct effect on labor productivity. More precisely, we assume that, by co-locating, firms increase their operating margins by reducing their fixed costs. In our case the degree of technological openness depends on how much of this reduction comes from local interaction and how much, instead, takes place across regional boundaries. Our assumption has the advantage of disentangling the effect of nonpecuniary knowledge spillover from the effect induced by the diversification of production. In other words, while previous NEG models essentially describe Jacobs' spillovers generating a variety-induced local advantage, our approach is more inspired to a Marshall-Arrow-Romer (MAR) kind of spillover in which local firms enjoy the technological advantage coming from a shared knowledge pool. In this respect, it could be useful to think to our assumption as a vertically integrated R\&D unit that improves productivity and whose efficiency increases due to external nonpecuniary spillovers from other R\&D units.

The analytical tractability of our model allows for the explicit derivation of geographical equilibria, defined as those distribution of firms where capital does not have incentives to change location. Our results present several differences with respect to the previous literature. In our case agglomeration is not due to the so called "home market effect," induced by the presence of regional differences in the exogenous endowment of factors. Rather, it is the endogenous self-reinforcing effect induced by technological externality that could be strong enough to generate a core-periphery $(\mathrm{CP})$ outcome. This implies that when knowledge spillovers are global, the only stable equilibrium is a symmetric distribution of economic activities between the two regions, irrespectively of the degree of trade openness. This should be confronted with the GS/LS models in Baldwin et al. (2003), where a sufficiently low transportation cost always implies a core-periphery outcome. At the same time, and differently from Martin and Rogers (1995) and the more recent Dupont and Martin (2006) where technological externalities are not considered, when trade costs are low enough and the economy is technologically segregated, agglomeration can emerge also with a priori identical locations. In general, increasing the flow of knowledge between regions reduces the total fixed costs, so that location choice is less relevant and agglomerated outcomes less likely. Ultimately, the equilibria of the economy and their desirability in terms of welfare are decided by the interplay between trade openness, as dependent on

\footnotetext{
${ }^{1}$ In early models, localized technological externalities were disposed off explicitly as sources of economic agglomeration, essentially because presumed to be particularly prone to measurement problems and modeling sloppiness (Krugman, 1991a, p. 53). 
transportation cost, and technological openness, as dependent on interregional spillovers. We show that the lower the technological barriers between the two regions, the larger the interval of transportation costs that lead to firms equidistribution.

We perform a complete welfare analysis. We find that the economic asymmetry induced by trade openness via profit seeking reallocation of capital can impoverish one region to the advantage of the other. If commercial integration is pushed further, since higher capital rents due to the technological externalities flow also to the periphery, welfare inequality is reduced. The initial loss of wealth, however, might hinder the implementation of trade opening policies. This effect can be mitigated, and possibly eliminated, if commercial and technological integrations are pursued together. We provide precise conditions under which either policy is to be preferred.

In Section 2, we introduce the model and derive the market equilibrium. In Section 3, we find the geographical equilibria of our economy and analyze their stability by studying how changes in the distribution of capital influence capital rents in both regions. In Section 4, we complete the characterization of geographical equilibria by making explicit their dependence on the parameters ruling trade and technological openness. The welfare analysis is presented in Section 5. In Section 6, we consider the case of a priori asymmetric regions and discuss how these asymmetries affect geographical equilibria. Section 7 concludes.

\section{THE MODEL}

Consider an economy with two locations, $l=1,2$, both populated by $L$ households ${ }^{2}$ so that $2 L$ is the total number of households. Each household is endowed with labor and capital and supplies them inelastically. The economy has a modern and a traditional sector. Whereas the traditional sector supplies a homogeneous good, the modern sector supplies differentiated products. In both sectors production is localized.

Households are "local" workers and "global" consumers, that is, they are immobile and work where they reside but they can buy goods produced in both locations. Households are also "global" investors, that is, they can supply capital to both locations. Modern goods are traded at a transportation cost that takes the form of an iceberg cost. We model it using an index of freeness of trade $\rho \in(0,1]$ : for one unit of the modern good to reach the other region, $1 / \rho$ units must be shipped. Traditional goods and capital are traded at no cost.

\section{Consumption}

All households have the same preferences and decide how much of the traditional good $C_{T}$ and of the bundle of modern goods $C_{M}$ to consume as to maximize a Cobb-Douglas utility function

$$
\mathrm{U}=\mathrm{C}_{T}^{1-\mu} \mathrm{C}_{M}^{\mu}
$$

with $\mu \in(0,1)$. As a result a fraction $\mu$ of each household income is spent on $C_{M}$ and a fraction $(1-\mu)$ is spent on $C_{T}$. The utility of the bundle $C_{M}$ is of CES type,

$$
\mathrm{C}_{M}=\left(\sum_{i=1}^{N} c_{i}^{\frac{\sigma-1}{\sigma}}\right)^{\frac{\sigma}{(\sigma-1)}} \quad \sigma>1,
$$

\footnotetext{
${ }^{2}$ We consider the case of unequally populated regions in Section 6 .
} 
with $c_{i}$ the consumption of good $i, i=1, \ldots, N$. This implies that the $N$ modern goods are substitutes, with a mutual elasticity of substitution equal to $\sigma$ (cfr. Dixit and Stiglitz, 1977).

\section{Production}

Each household is endowed with one unit of labor, and there is not an a priori distinction between workers of the modern and traditional sector. The traditional sector uses labor as the unique input under constant returns to scale with unitary marginal costs. Due to the large number of potential producers, as we shall see at least $2 L(1-\mu)$ at equilibrium, this market is perfectly competitive and the traditional good is sold at its marginal cost, which we take as the numeràire of the economy.

Both capital and labor are used in the production of the $N$ modern goods. The amount of labor $v_{i}$ that firm $i=1, \ldots, N$ employs to produce an amount $y_{i}$ of the modern output is given by the scale economy cost function

$$
v_{i}=\beta y_{i}+\alpha_{l_{i}},
$$

where $\beta$ is constant across firms and across locations, and $\alpha_{l_{i}}$ is the fixed amount of labor necessary to start production. Whereas the marginal productivity of labor is assumed to be constant and equal in the two locations, the fixed amount of labor might depend on the location $l_{i}$ of firm $i$. We shall assume that $\alpha_{l_{i}}$ is a function of firms' location, as stated below. Each firm also needs one unit of capital, available at the price $r_{i}$. This, at market equilibrium, is given by the operating profits

$$
r_{i}=p_{i} y_{i}-w_{i} v_{i}
$$

where $p_{i}$ is the price of good $i$ and $w_{i}$ is the cost of labor for firm $i$.

Given the structure of preferences in (2) each firm produces a different product. The total number of varieties produced in each location is thus equal to the amount of capital available there.

Each household is endowed with the same amount of capital $N / 2 L .^{3}$ Assuming that households maximize their capital revenues, and since capital is moved without costs, their investment choices are symmetric so that each household invests a fraction $1 / 2 L$ of capital in each firm. ${ }^{4}$ The market structure is that of monopolistic competition, that is, each firm maximizes its profits given market demand elasticity and irrespectively of other firms behaviors.

\section{Technological Externality}

So far our assumptions closely mimic footloose capital models, such as Martin and Rogers (1995) or Dupont and Martin (2006). Departing from these works, we introduce a localized technological externality, which we model as a term of direct firms interaction not mediated by market forces. More specifically we assume that the required fixed amount of labor $\alpha_{l}$ decreases with the number of firms located in a region according to

$$
\alpha_{l}=\alpha\left(n_{l}\right)=\frac{\alpha}{\frac{n_{l}}{N}+\lambda\left(1-\frac{n_{l}}{N}\right)},
$$

\footnotetext{
${ }^{3}$ In our model, the global amount of capital, $N$, is an exogenous variable. Notice, however, that $N$ is not entirely free, see also Lemma 1 and 2.

${ }^{4}$ This is usually assumed in footloose capital models (see Baldwin et al., 2003, p. 74) to avoid the complications resulting from household strategic interaction. The assumption is harmless at a geographical equilibrium, that is, at a distribution of firms where either rents in both locations are equal or all firms are in the same location. The assumption is, however, not harmless out of equilibrium and stability results do in general depend on it.
} 
where $n_{l}$ is the number of firms producing modern goods in location $l \in\{1,2\}$. Equation (5) represents a positive localized externality because the presence of more local producers decreases the production cost. The marginal decrease of fixed costs, or increase of internal $R \& D$ efficiency, is dependent on the interregional spillover parameter $\lambda$, representing the technological openness of the whole economy. The functional specification is analogous to the interregional spillover in R\&D activity used by Martin (1999), Martin and Ottaviano (1999), Baldwin and Forslid (2000). The difference between that literature and the present work is that we do not model a R\&D sector and a local market for patents, but instead assume that each firm operates an internal R\&D unit. The interfirm knowledge spillover improves firm efficiency by generating a reduction of fixed costs. This reduction increases proportionally with the number of firms, and thus R\&D units, located in the same region. From a theoretical standpoint, the assumption of a labor-enhancing knowledge spillover, and, to some extent, the vertical integration of $R \& D$ and production activities, seems in line with the widespread idea that the tacit component of knowledge, which is essential to its localized nature, represents a contribution to human capital and is thus embedded in the labor factor.

The effect of interregional knowledge spillover can be easily understood from (5). For low values of $\lambda$ the economy splits in technological separated parts and firms can exclusively exploit advantages derived by colocation in the same region. When $\lambda=0$ research and development in the two locations are completely segregated and the total fixed cost to be paid in each location is constant and equal to $N \alpha$. Unless the modern sector is entirely agglomerated in one location, each firm pays more than $\alpha$ in labor fixed costs. For positive values of $\lambda$, conversely, the economy is technologically integrated, and productivity improving positive externalities are also operating across regions. The higher the value of $\lambda$, the stronger the interregional effect. Notably, the existence of interregional spillovers impacts on fixed costs in two ways. First, it creates a global advantage by reducing production costs for all firms, thus increasing modern sector profits. Second, it makes location choice less relevant and uneven outcomes less likely. In the extreme case of $\lambda=1$, technological externalities operate across all firms and each firm pays the same fixed cost $\alpha$, irrespectively of its location.

\section{Market Equilibria}

Due to zero transportation costs, the price of the traditional good must be the same in both locations. Moreover, because of perfect competition, constant returns to scale, and unitary labor productivity, the wage of workers in the traditional sector is equal to the price of the traditional good, our numeràire. Given that workers are not mobile, at equilibrium it should be indifferent to work in the traditional or modern sector. As a result, wages in both sectors are equal to one.

Location by location, firms produce using the same technology, face the same demand, and the same labor supply. This implies that equilibrium prices, quantities, and wages are the same for all the firms in a given location.

To derive market equilibria we proceed as follows: Exploiting the CES preference structure (2), we compute consumer demand for the goods produced in each location. We consider that all goods are substitutes, that transportation costs impact the consumption of foreign goods, and that the budget constraint depends also on the capital rent. From the monopolistic competition structure of the market, and knowing consumers demand elasticity, we derive firms pricing behavior. By setting supply equal to demand we are able to determine equilibrium quantities and capital rents as a function of firms distribution across locations. The following step is to use capital rents and labor income to determine consumers demand for the traditional good. Hence, we can derive the traditional good 
required supply and the labor needed to produce it. As a result, we can derive the total labor demand. Next, we have to check that the labor market is at equilibrium too. Since there are two segmented labor markets, requiring that both clear amounts to posing a constraint on agents preferences and on the scale of the economy. Finally, we have to impose that capital rents are positive, or otherwise households do not have any incentive to spent their capital endowment.

Let us start from consumers demand. Denote the quantity consumed by a consumer who resides in $l$ of a product produced in $m$ as $d_{l m}$ with $l, m \in\{1,2\}$. Relative demand in location $l$ under CES utility satisfies

$$
\frac{d_{l l}}{d_{l m}}=\left(\frac{p_{m}}{\rho p_{l}}\right)^{\sigma}, \quad m \neq l
$$

Agents budget constraint in $l$ is

$$
\mu I\left(n_{1}, n_{2}\right)=n_{l} d_{l l} p_{l}+n_{m} d_{l m} \frac{p_{m}}{\rho}, \quad m \neq l,
$$

where $I\left(n_{1}, n_{2}\right)=1+R\left(n_{1}, n_{2}\right)$ is the household income, given by her unitary wage plus her share of capital rent, $R\left(n_{1}, n_{2}\right)$, yet to be found. Using the previous equations to find the demand of local and foreign goods in $l$ leads to

$$
d_{l l}=\frac{\mu I\left(n_{1}, n_{2}\right)}{n_{l} p_{l}+n_{m} p_{l}^{\sigma} p_{m}^{1-\sigma} \rho^{\sigma-1}}, \quad d_{l m}=\frac{\mu I\left(n_{1}, n_{2}\right) \rho^{\sigma}}{n_{l} p_{l}^{1-\sigma} p_{m}^{\sigma}+n_{m} p_{m} \rho^{\sigma-1}}, \quad m \neq l .
$$

Given the market structure of monopolistic competition each firm, knowing consumers inverse demand, sets its output so that marginal revenues are equal to marginal costs. In each location $l$ this gives

$$
p_{l}\left(1+\frac{1}{\varepsilon}\right)=\beta
$$

where $\varepsilon=\partial \log c / \partial \log p$ is the demand elasticity, and we have used the fact that wages are equal to one. Given (2), as long as the number of commodities $N$ is large (see Dixit and Stiglitz, 1977, for the details), it holds that $\varepsilon=-\sigma$, which together with (9) implies $p_{l}=\beta \frac{\sigma}{\sigma-1}$.

Let $y_{l}$ denotes the output of a firm producing a modern good in $l$. Equating, location by location, supply and demand, we get

$$
y_{l}=L d_{l l}+L \frac{d_{m l}}{\rho}, \quad m \neq l
$$

Using the demand derived in (8) and substituting the expression for prices, we can easily solve for market equilibrium quantities. After introducing the rescaled freeness of trade parameter $\phi=\rho^{\sigma-1}$, we get

$$
y_{l}=\mu I\left(n_{1}, n_{2}\right) L \frac{\sigma-1}{\beta \sigma}\left(\frac{1}{n_{l}+n_{m} \phi}+\frac{\phi}{n_{l} \phi+n_{m}}\right), \quad m \neq l .
$$

The expression above and that for prices can be used to derive from (4) and (5) firms capital rents in both locations. Letting $x=n_{1} / N$ be the fraction of firms (i.e., capital) in 
location 1 , so that $n_{2}=(1-x) N$, capital rents can be written as ${ }^{5}$

$$
\left\{\begin{array}{l}
r_{1}(x)=\frac{\mu I(x) L}{N \sigma}\left(\frac{1}{x+(1-x) \phi}+\frac{\phi}{x \phi+(1-x)}\right)-\frac{\alpha}{x+\lambda(1-x)}, \\
r_{2}(x)=\frac{\mu I(x) L}{N \sigma}\left(\frac{1}{x \phi+(1-x)}+\frac{\phi}{x+(1-x) \phi}\right)-\frac{\alpha}{1-x+\lambda x} .
\end{array}\right.
$$

Notice that this is still an implicit equation because $I(x)$ is a function of $r_{1}(x)$ and $r_{2}(x)$. In fact, remembering that each households invests a fraction $1 / 2 L$ of capital in each firm, it holds

$$
I(x)=1+\frac{N}{2 L}\left(x r_{1}(x)+(1-x) r_{2}(x)\right) .
$$

Solving (12) for $r_{1}(x)$ and $r_{2}(x)$, we get

$$
I(x)=1+R(x)=1+\frac{\mu-\frac{N}{2 L} \sigma \alpha\left(\frac{x}{x+\lambda(1-x)}+\frac{1-x}{1-x+\lambda x}\right)}{(\sigma-\mu)} .
$$

Equations (12) and (13) define a market equilibrium provided that, location by location, the modern sector firms' labor demand is always smaller than $L$. The condition will be imposed in the following where we summarize our findings:

LEMMA 1. Given the scale of the economy $S=N / L$, if it holds

$$
S<\widetilde{S}=\frac{\mu+\sigma-2 \mu \sigma}{\alpha \sigma(1-\mu)},
$$

then for any geographical distribution $x \in[0,1]$ the global market for the traditional good, the global markets for the $N$ modern goods, and the two local labor markets clear. Location rents and household income are given by (12) and (13), respectively.

Finally, to ensure that households do actually invest in the modern sector we impose that the per-capita rent of the economy is positive. We have the following lemma:

LEMMA 2. The per-capita rent of the economy $R(x)$ is positive for any value of the freeness of trade $\phi$ and of the interregional spillover $\lambda$ provided that

$$
S=\frac{N}{L}<\bar{S}=\frac{\mu}{\alpha \sigma} .
$$

In what follows, we shall assume that the scale $S$ is such that both constraints in (14) and (15) hold, which is equivalent to assume that $S<\min \{\widetilde{S}, \bar{S}\} .{ }^{6}$ Apart from the foregoing condition, our analysis is valid for any exogenously defined number of firms (units of capital) $N$. In particular, the condition can only be met when $\widetilde{S}>0$, or, in terms of the preference for the modern goods, $\mu<\sigma /(2 \sigma-1)$. The share of income spent on modern goods should not be too big. ${ }^{7}$ Summing up, for any given elasticity of substitution, provided that preferences for modern goods are not too strong, there always exists a range

\footnotetext{
$[0,1]$.

${ }^{5}$ Throughout the paper, without loss of generality, we consider $x$ to be a real number in the interval

${ }^{6}$ By simple computations one can show that $\bar{S}=\min \{\widetilde{S}, \bar{S}\}$ when $\mu \in[0,1 / 2]$ or when $\mu \in(1 / 2,1]$ and $\sigma<\mu^{2} /(2 \mu-1)$, and $\widetilde{S}=\min \{\widetilde{S}, \bar{S}\}$ otherwise.

${ }^{7}$ This is the same condition on consumers preferences found in, e.g., Forslid and Ottaviano (2003). Notice that the condition is always satisfied when $\mu \leq 1 / 2$.
} 
of firms-to-households ratios such that markets in both locations are at equilibrium and capital rents are nonnegative.

The dependence of equilibrium capital rents on the geographical distribution of firms is due to both pecuniary and technological externalities. The effect of the former goes via the sum of local and foreign demand, the part of capital rents in (12) that depends on $\phi$. When concentration of local firms is low, each firm faces a high local demand and makes high profits. As the concentration of local firms increases, a higher competition lowers profits coming from the local demand which, due to positive transportation costs, are not fully compensated by an increased foreign demand. Thus, pecuniary externalities have a negative effect on agglomeration. The effect becomes stronger as transportation costs increase, i.e., freeness of trade decreases.

Technological externalities influence equilibrium capital rents both locally and globally. The local effect is due to the direct dependence of fixed costs on the geographical distribution of firms, as given by the last term of both expressions in (12). The higher the concentration of firms in location $l$, the lower the fixed costs and the higher the capital rent of firms located there. The global effect is due to the dependence of local rents in (12) on global capital rents $R(x)$ and acts as a multiplier. In fact, the geographical distribution has first an impact on total fixed costs, and thus on total capital rents, which, in turn, have a wealth effect on consumers demand, thus affecting capital rent in each location. An increase in the concentration of firms lowers total fixed costs payed by all firms, increases total capital rents, increases households wealth, increases total demand and, in turn, increases capital rents further. Notice that whereas the local effect increases the rent in a given location through local agglomeration, the global effect increases capital rents in both locations, no matter where firms do actually agglomerate. The overall effect of these two forces and their strength depend both on the interregional knowledge spillover $\lambda$ and on the freeness of trade $\phi$. In general, when $\lambda$ is low (high) the technological externality is (not) localized and its variability with local concentration is high (low). In the extreme case, $\lambda=1$, both regions have equal benefits, irrespectively of the geographical distribution of firms.

\section{GEOGRAPHICAL EQUILIBRIA}

We assume that capital moves from one location to the other following the rent difference $\Delta(x)=r_{1}(x)-r_{2}(x)$. When $\Delta(x)$ is positive capital flows from location 2 to location 1 , the other way round when $\Delta(x)$ is negative. The geographical equilibria can be derived looking at the fixed point of the following generic dynamical system

$$
\frac{d x}{d t}= \begin{cases}\max \{0, \Delta(x)\} & \text { if } x=0, \\ F(\Delta(x)) & \text { if } 0<x<1, \\ \min \{0, \Delta(x)\} & \text { if } x=1,\end{cases}
$$

where $F$ is a strictly increasing differentiable function, $F^{\prime}(\Delta(x))>0$, with $F(0)=0$. Despite different functions $F$ correspond to different trajectories, the interior fixed points of the dynamics in (16) are the distributions $x$ having zero rent difference, the solutions of $\Delta(x)=0$, and their local stability is determined by the sign of their marginal rent difference $\Delta^{\prime}(x)$. The definition of the dynamical system at the border is due to the fact that the variable $x$ is constrained to be in the interval $[0,1]$ so that 0 and 1 are other possible fixed points, depending on the sign of rent difference when all firms are located in the same region, $\Delta(0)$ and $\Delta(1)$. We have the following definition:

DEFINITION 1. An interior geographical equilibrium $x \in(0,1)$ is an asymptotically stable interior fixed point of (16). A border geographical equilibrium $x \in\{0,1\}$ is a 
asymptotically stable border fixed point of (16), that is, $x=0$ is a border geographical equilibrium if $\lim _{x \rightarrow 0^{+}} \Delta(x)<0$, and $x=1$ is a border geographical equilibrium if $\lim _{x \rightarrow 1^{-}} \Delta(x)>0$.

We shall name an interior geographical equilibrium a nonagglomerated economy (NAG) when $x=1 / 2$, and a partially agglomerated economy (PAG) when $x \neq 1 / 2$. An agglomerated economy $(A G)$ is observed when at least one border is a geographical equilibrium.

Let us start from the existence of geographical equilibria corresponding to NAG and AG. Given the symmetry of the economy it always holds that rents are equal at $x=1 / 2$ so that $\Delta(1 / 2)=0$. As a result, when the marginal rent difference $\Delta^{\prime}(1 / 2)$ is negative, $x=1 / 2$ is asymptotically stable and NAG occurs upon having an initial condition close enough to $x=1 / 2$. The same symmetry also implies that the sign of the rent difference when all firms are in $l=2, \Delta(0)$, is the opposite of the sign of the rent difference when all firms are in $l=1, \Delta(1)$. It follows that either both or none of the two borders are geographical equilibria. In particular, AG occurs when $\Delta(0)$ is negative.

NAG and AG are not the only possible long-run outcomes. There might be other interior fixed points leading to PAG. Importantly, it turns out that, given our formulation of the technological externality, the sign of the rent difference when all firms are agglomerated in one location and the sign of the marginal rent difference when firms are distributed evenly across the two locations are enough to characterize the existence and the stability of all the geographical equilibria.

PROPOSITION 1. Assume $\widetilde{S}>0$ and $S<\min \{\widetilde{S}, \bar{S}\}$. Define $x^{-}, x^{+}$as

$$
x^{ \pm}=\frac{1}{2}\left(1 \pm \sqrt{\frac{a}{a+2 b}}\right) \text {, }
$$

where

$$
\begin{aligned}
& a=(1-\phi)^{2}\left(\alpha N \sigma(1-\lambda)-\mu L(1+\lambda)^{2}+2 \alpha N \mu \lambda\right)+4 \alpha N \phi(1-\lambda)(\sigma-\mu), \\
& b=\mu \lambda(1-\phi)^{2}(2 L-\alpha N)-2 \alpha N \phi(1-\lambda)(\sigma-\mu) .
\end{aligned}
$$

Consider the trajectories of the dynamical system (16) given the initial condition $x(t=0)=$ $x_{0}$. It holds that

- if $\Delta(0)=\Delta^{\prime}(1 / 2)=0$, all $x \in[0,1]$ are stable, but not asymptotically stable, fixed points of (16). In this case, there are no geographical equilibria.

- if $\Delta(0) \leq 0$ and $\Delta^{\prime}(1 / 2) \geq 0$, but $\Delta(0) \neq 0$ or $\Delta^{\prime}(1 / 2) \neq 0$, only AG occurs. The geographical equilibrium is either 0 or 1 depending on initial conditions, the former when $x_{0}<1 / 2$ the latter when $x_{0}>1 / 2$.

- if $\Delta(0) \geq 0$ and $\Delta^{\prime}(1 / 2) \leq 0$, but $\Delta(0) \neq 0$ or $\Delta^{\prime}(1 / 2) \neq 0$, only NAG occurs. The geographical equilibrium is $1 / 2$ irrespectively of the initial condition.

- if $\Delta(0)<0$ and $\Delta^{\prime}(1 / 2)<0$ both NAG and $A G$ occur. The geographical equilibrium is 0 when $x_{0}<x^{-}, 1 / 2$ when $x_{0} \in\left(x^{-}, x^{+}\right)$, and 1 when $x_{0}>x^{+}$.

- if $\Delta(0)>0$ and $\Delta^{\prime}(1 / 2)>0$ only $P A G$ occur. The geographical equilibrium is $x^{-}$when $x_{0} \in[0,1 / 2)$, and $x^{+}$when $x_{0} \in(1 / 2,1]$.

The results of the previous proposition are summarized in Figure 1. Specific examples of the functional form of the rent difference $\Delta(x)$ are given in Figure 2. Apart from the nongeneric case when both $\Delta(0)$ and $\Delta^{\prime}(1 / 2)$ are zero, there are at most five different geographical equilibria, the two border equilibria 0 and 1 and the three interior equilibria $1 / 2, x^{+}$, and $x^{-}$. 


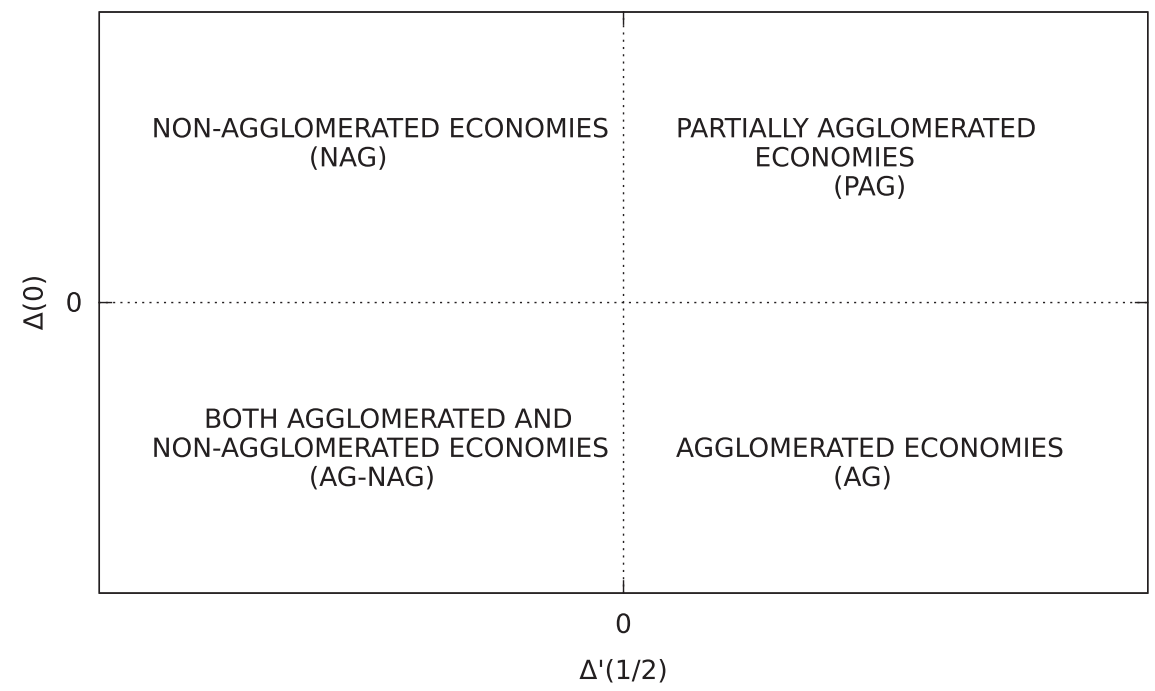

FIGURE 1: Existence of Different Types of Geographical Equilibria in the Plane $\left(\Delta^{\prime}(1 / 2), \Delta(0)\right)$.
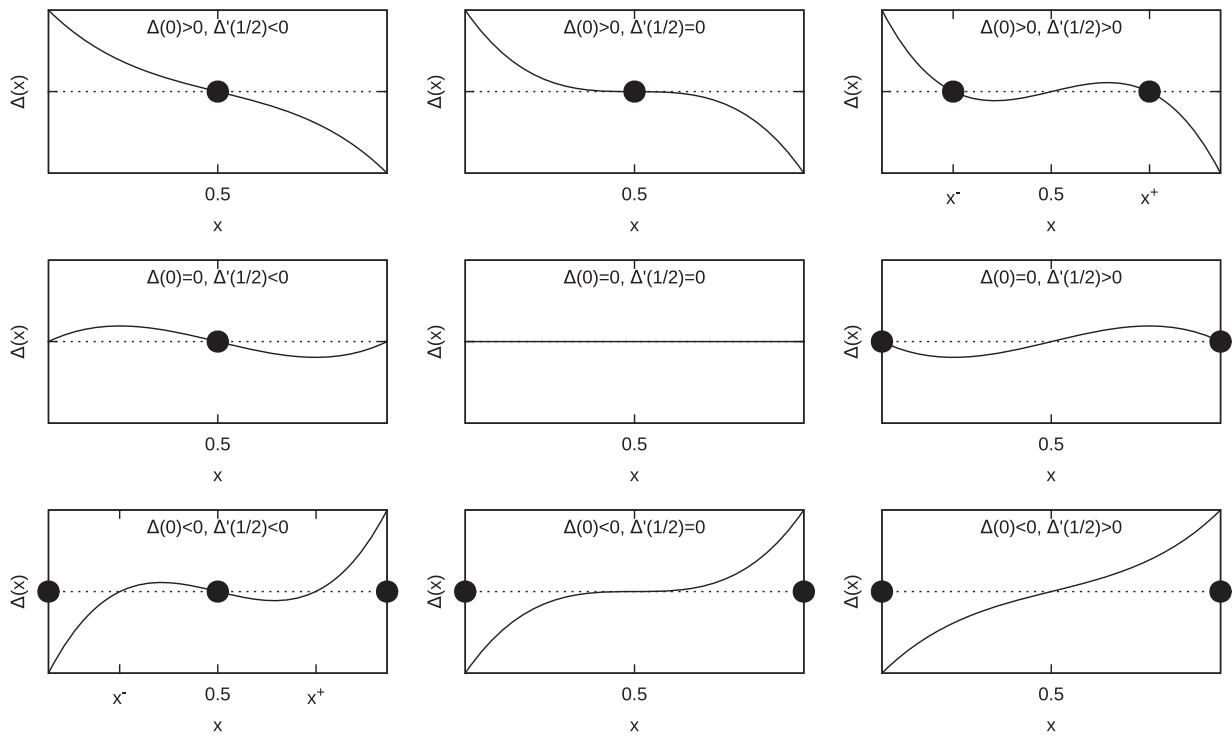

Note: Geographical equilibria are represented by black dots.

FIGURE 2: Capital Rent Differences $\Delta(x)$ for All the Sign Combinations of the Coefficients $\Delta^{\prime}(1 / 2)$ and $\Delta(0)$.

The two interior fixed points $x^{+}$and $x^{-}$exist only when the marginal rent difference at $1 / 2, \Delta^{\prime}(1 / 2)$, and the rent difference of an agglomerated economy, $\Delta(0)$, have the same sign. If this sign is positive, these fixed points are geographical equilibria. In this case, a complete agglomeration in $l=2$ generates higher rents in $l=1$ and firms move there until the rent difference becomes zero. Since $\Delta^{\prime}(1 / 2)>0$ the zero rent difference must occur at $x^{-}<1 / 2$. The same happens starting from a complete agglomeration in $l=1$. The 
economy moves toward an interior equilibrium with a share of firms equal to $x^{+}>1 / 2$. The basin of attraction of the internal equilibria is easily derived: if $x_{0}>1 / 2$ the economy moves toward $x^{+}$. Otherwise it moves toward $x^{-}$.

Conversely if the sign of $\Delta^{\prime}(1 / 2)$ and $\Delta(0)$ are both negative, the two internal fixed points $x^{ \pm}$are unstable. In this case AG and NAG equilibria coexist. If the initial condition is $x_{0} \in\left(x^{-}, x^{+}\right)$, the system converges to the nonagglomerated case $x=1 / 2$. Otherwise, the systems converges toward a fully agglomerated economy, with all firms in location 1 if $x_{0}>x^{+}$or all firms in location 2 if $x_{0}<x^{-}$.

When $\Delta(0)$ and $\Delta^{\prime}(1 / 2)$ have opposite signs, only one type of equilibria exists. If $\Delta(0)>0$ the NAG equilibrium is a global attractor, that is the economy converges toward $x=1 / 2$ irrespectively of the initial condition. If $\Delta(0)<0$ the economy ends up in one of the two AG equilibria: all firms concentrated in location 1 if $x_{0}>1 / 2$, or in location 2 if $x_{0}<1 / 2$.

Proposition 1 also shed lights on the possible transitions between the different type of geographical equilibria as changes in the parameters of the economy occur. We analyze the five different possible transitions (both coefficients are equal to zero, one of the two is equal to zero while the other is positive, the same when the other is negative) with the help of Figure 2 . When $\Delta(0)$ is positive and $\Delta^{\prime}(1 / 2)$ changes sign from negative to positive, the economy transits from NAG to PAG. In particular, when $\Delta^{\prime}(1 / 2)=0$ NAG occurs. Otherwise, when $\Delta(0)$ is negative and $\Delta^{\prime}(1 / 2)$ changes sign, AG always occurs while NAG occurs for $\Delta^{\prime}(1 / 2)<0$ and vanishes otherwise. Using the language of bifurcation theory, the two phenomena are known, respectively, as a subcritical and supercritical pitchfork bifurcation. Importantly, in the former case the transition between nonagglomeration and agglomeration is smooth and does not exhibit the typical hysteresis phenomenon associated with the latter. The same type of argument is valid when $\Delta(0)$ changes sign. In this case, the transition between full agglomeration and nonfull agglomeration exhibits hysteresis when $\Delta^{\prime}(1 / 2)<0$, and it is smooth otherwise.

\section{THE EFFECT OF TRADE AND TECHNOLOGICAL OPENNESS}

In this section, we are interested in the influence of interregional spillovers and freeness of trade on the possible geographical equilibria of the economy. We assume consumer preferences, costs structures, number of firms, and households as given and subject to the two restrictions (14) and (15). We then translate the geographical equilibria conditions of Proposition 1 in terms of the more directly interpretable policy parameters $\lambda$ and $\phi$. This is the content of the following proposition:

PROPOSITION 2. Assume $\widetilde{S}>0$ and $S<\min \{\widetilde{S}, \bar{S}\}$. The functions

$$
\begin{aligned}
& \phi^{a}(\lambda)=\left(1+\Gamma(\lambda)-\sqrt{\Gamma^{2}(\lambda)+2 \Gamma(\lambda)}\right)^{\frac{1}{\sigma-1}}, \\
& \phi^{b}(\lambda)=\left(1+\Theta(\lambda)-\sqrt{\Theta^{2}(\lambda)+2 \Theta(\lambda)}\right)^{\frac{1}{\sigma-1}},
\end{aligned}
$$

with

$$
\begin{aligned}
\Gamma(\lambda) & =\frac{\alpha N(1-\lambda)(\sigma-\mu)}{\mu \lambda(2 L-\alpha N)+\frac{1-\lambda}{2}(\mu L(1-\lambda)-N \alpha \sigma)}, \\
\Theta(\lambda) & =\frac{\alpha N(1-\lambda)(\sigma-\mu)}{\mu \lambda(2 L-\alpha N)}
\end{aligned}
$$




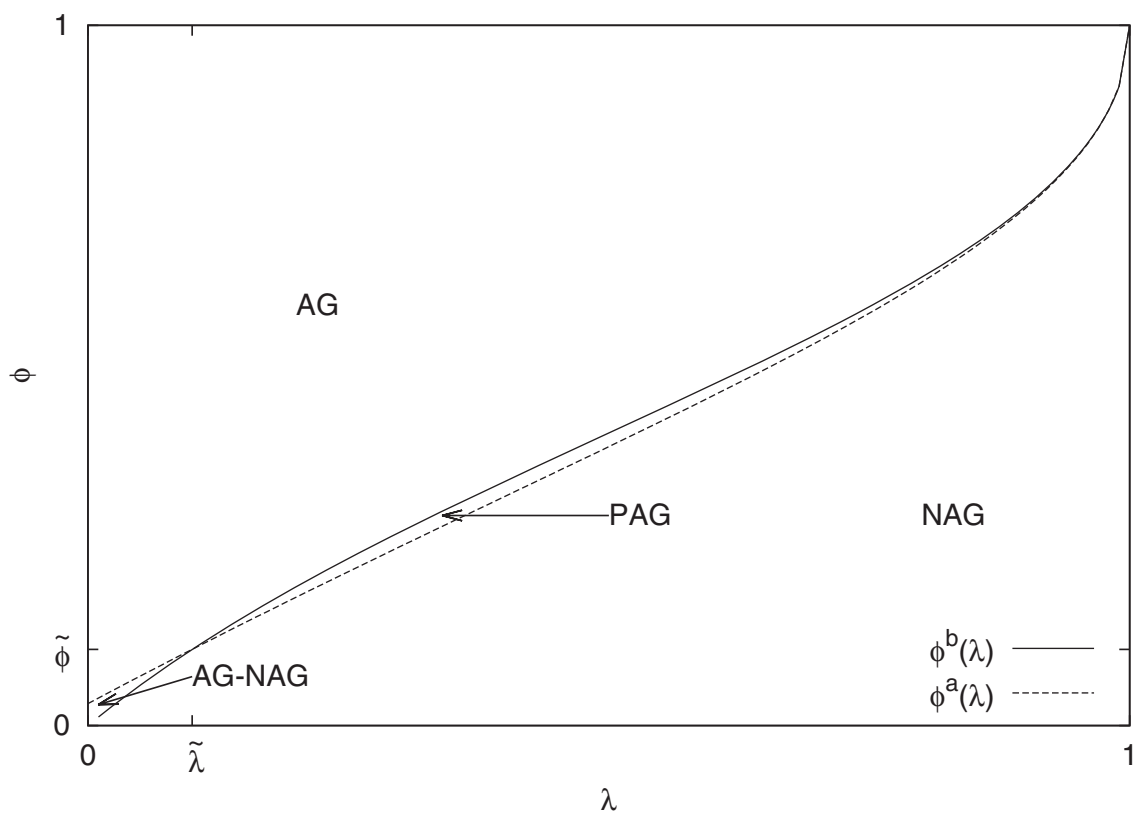

Note: The values of the economy parameters are $L=400, N=150, \sigma=3, \mu=0.5, \alpha=0.4$.

FIGURE 3: Existence of Different Types of Geographical Equilibria in the Plane $(\lambda, \phi)$. map the interval $(0,1]$ in the interval $(0,1]$ such that

$$
\begin{aligned}
\Delta^{\prime}(1 / 2) & \gtreqless 0 \Leftrightarrow \phi \gtreqless \phi^{a}(\lambda), \\
\Delta(0) & \gtreqless 0 \Leftrightarrow \phi \lesseqgtr \phi^{b}(\lambda) .
\end{aligned}
$$

Moreover it holds $\phi^{b}(1)=\phi^{a}(1)=1, \phi^{b}(0)=0$ and, when $\lambda \gtreqless \tilde{\lambda}=1-S / \bar{S}$, it is $\phi^{b}(\lambda) \gtreqless$ $\phi^{a}(\lambda)$.

Figure 3 illustrates the results of Proposition 2. It shows for which values of the policy parameters the three types of equilibria, AG, NAG, or PAG, are observed. When trade is less free, $\phi<\min \left\{\phi^{a}(\lambda), \phi^{b}(\lambda)\right\}$, the pecuniary externality dominates and the outcome is NAG. Irrespectively of the initial geographical distribution, capital, and thus firms, distribute equally between the two regions. When trade barriers are low, $\phi>\max \left\{\phi^{a}(\lambda), \phi^{b}(\lambda)\right\}$, the technological externality dominates, agglomeration on either sides is a geographical equilibrium and the long-run dynamics converges either to 0 or 1 depending on initial conditions.

When the freeness of trade takes intermediate values, geographical equilibria of different kind can coexist and two different scenarios are possible. For low interregional spillovers, i.e., when $\lambda<\tilde{\lambda}$, if $\phi \in\left(\phi^{b}(\lambda), \phi^{a}(\lambda)\right)$ the economy can be either in NAG or AG configuration (c.f. the left panel of Figure 4). In this case, the transition from NAG to AG, due to the progressive opening-up of the economy, is abrupt. Moreover, the economy shows hysteresis, that is, once the transition has occurred it cannot be reverted by decreasing the freeness of trade.

The scenario is different when the interregional spillovers are higher, i.e., $\lambda>\tilde{\lambda}$. As shown in the right panel of Figure 4, in this case AG and NAG are still associated, respectively, with high and low values of $\phi$, but the transition between the two equilibria is smoother. Indeed, for intermediate values of $\phi$, between $\phi^{a}(\lambda)$ and $\phi^{b}(\lambda)$, two asymmetric 

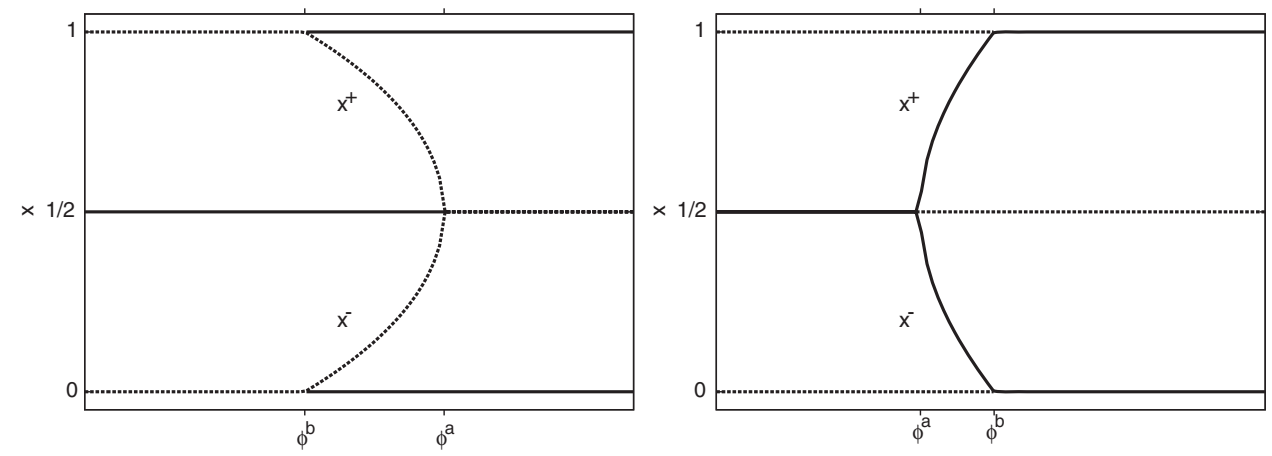

Note: Continuous lines denote geographical equilibria. Left panel: $\lambda<\tilde{\lambda}$. Right panel: $\lambda>\tilde{\lambda}$.

FIGURE 4: Internal Fixed Points and Boundary Distributions as a Function of $\phi$.

geographical equilibria emerge, collapsing on the border (interior symmetric) equilibria as $\phi$ increases (decreases). These distributions represent PAG configurations: due to local spillover, the partial concentration of modern goods production is advantageous but, due to relatively high transportation costs, a further agglomeration is not beneficial as would only increase competition in the crowded location without enough profits coming from an increased demand in the other region.

Despite in Figure 3 the curves $\phi^{a}(\lambda)$ and $\phi^{b}(\lambda)$ are plotted for specific values of the economy parameters $(L, N, \mu, \sigma$, and $\alpha$ ), their behavior and, in particular, the regions they identify are general properties of the model. Indeed, it always holds that $\phi^{b}(0)=$ $0, \phi^{b}(1)=\phi^{a}(1)=1, \phi^{b}(\tilde{\lambda}) \gtreqless \phi^{a}(\tilde{\lambda})$ when $\lambda \gtreqless \tilde{\lambda}$, and $\tilde{\lambda} \in(0,1)$. For the same reason the bifurcation phenomena illustrated in Figure 4 are also general.

Notice at last that the closer $S$ is to $\bar{S}$, that is the higher the scale of the economy and the lower the capital profits, the closer $\tilde{\lambda}$ is to 0 , so that the smooth transition from NAG to AG occurs for a larger set of policy parameters values.

In summary, the set of dynamics displayed by our model is relatively rich. The nature of the transition between nonagglomerated and core-periphery equilibria depends on technological openness. ${ }^{8}$ When the share of knowledge spilling across regional boundaries is low, progressive trade globalization can lead to the catastrophic agglomeration of the entire modern sector in one location. In this case, the economy displays hysteresis effect, typical of NEG models, which locks the economy in a core-periphery equilibrium also if higher trade costs are reintroduced. Conversely, if the interregional knowledge spillovers are strong enough, there exists a smooth equilibrium transition between equidistribution and agglomeration, with partial agglomeration for intermediate values of transportation cost. In this case an opening of interregional trade does not imply an abrupt reallocation of economic activities and the hysteresis effect is absent.

\section{WELFARE ANALYSIS}

So far we have assumed that capital moves in order to maximize its rent, rather than households real income. This begs the question of what happens to household utilities, that is, to their welfare level. Household's utility in each location can be written as total

\footnotetext{
${ }^{8}$ The result should be confronted with the LS model in Baldwin et al. (2003) where the transition is always smooth as long as the technological integration is not complete.
} 
income divided by the price index

$$
\left\{\begin{array}{l}
W_{1}(x)=\frac{I(x)}{P_{1}(x)} \\
W_{2}(x)=\frac{I(x)}{P_{2}(x)}
\end{array}\right.
$$

where $^{9}$

$$
P_{1}(x)=\left(\frac{\beta \sigma}{\sigma-1}\left(N x+N(1-x) \rho^{\sigma-1}\right)^{\frac{-1}{\sigma-1}}\right)^{\mu},
$$

and $P_{2}(x)=P_{1}(1-x)$. The properties of $I(x)$ and $P_{1,2}(x)$ (c.f. the Proof of Proposition 3) imply that the welfare difference

$$
\Delta W(x)=I(x) \frac{P_{2}(x)-P_{1}(x)}{P_{1}(x) P_{2}(x)}
$$

is negative in $x=0$, zero only in $x=1 / 2$, and positive in $x=1$. As a result, each household is better off if firms agglomerate in his/her own region. In this case, local households do not pay transportation costs for modern goods and, because of technological externalities, their income benefits from the strongest possible abatement of fixed costs.

Whereas agglomeration is clearly beneficial for the region that happens to host the modern sector, it is not clear whether it is beneficial also for the whole economy. This is an important issue in a model like ours, where regions are ex ante identical and where workers are not mobile. Finding an answer requires to investigate what happens to the welfare of the region that specializes in the traditional sector. On the one hand, households living there have to import modern goods so that, due to transportation costs, they have higher real prices. On the other hand, their nominal income is the same as that of households located in the modern region, and they also profit from higher capital rents. The overall result depends on the relative strength of these two effects, which in turn is related to both trade costs and interregional spillovers, that is, to market and technological openness.

Welfare analysis clearly depends on which type of welfare aggregating function one considers. Since we are mainly concerned with welfare levels in the traditional region, the $\max -\min$ formulation seems the most appropriate. We define total welfare to be equal to the minimal welfare level between the two regions:

$$
W_{T}(x)=\min \left\{W_{1}(x), W_{2}(x)\right\} .
$$

Given that benefits of agglomeration spill also to the traditional region, it may well be the case that agglomeration is the best outcome, also under such an egalitarian definition of total welfare. The following proposition states when this is the case.

PROPOSITION 3. Consider total welfare as in (22). For any given value of the interregional spillover $\lambda$, provided that freeness of trade $\rho$ is such that

$$
\rho>\rho^{w}(\lambda)=\left(2\left(\frac{L-\frac{N \alpha}{2}}{L-\frac{N \alpha}{1+\lambda}}\right)^{\frac{\sigma-1}{\mu}}-1\right)^{-\frac{1}{\sigma-1}},
$$

AG is the global welfare maximum. Otherwise, when $\rho<\rho^{w}(\lambda)$, NAG is a global maximum. When $\rho=\rho^{w}(\lambda)$ both NAG and AG are global maxima.

\footnotetext{
${ }^{9}$ In this section, we prefer $\rho$ to $\phi$ because it does not depend on the preference parameter $\sigma$.
} 


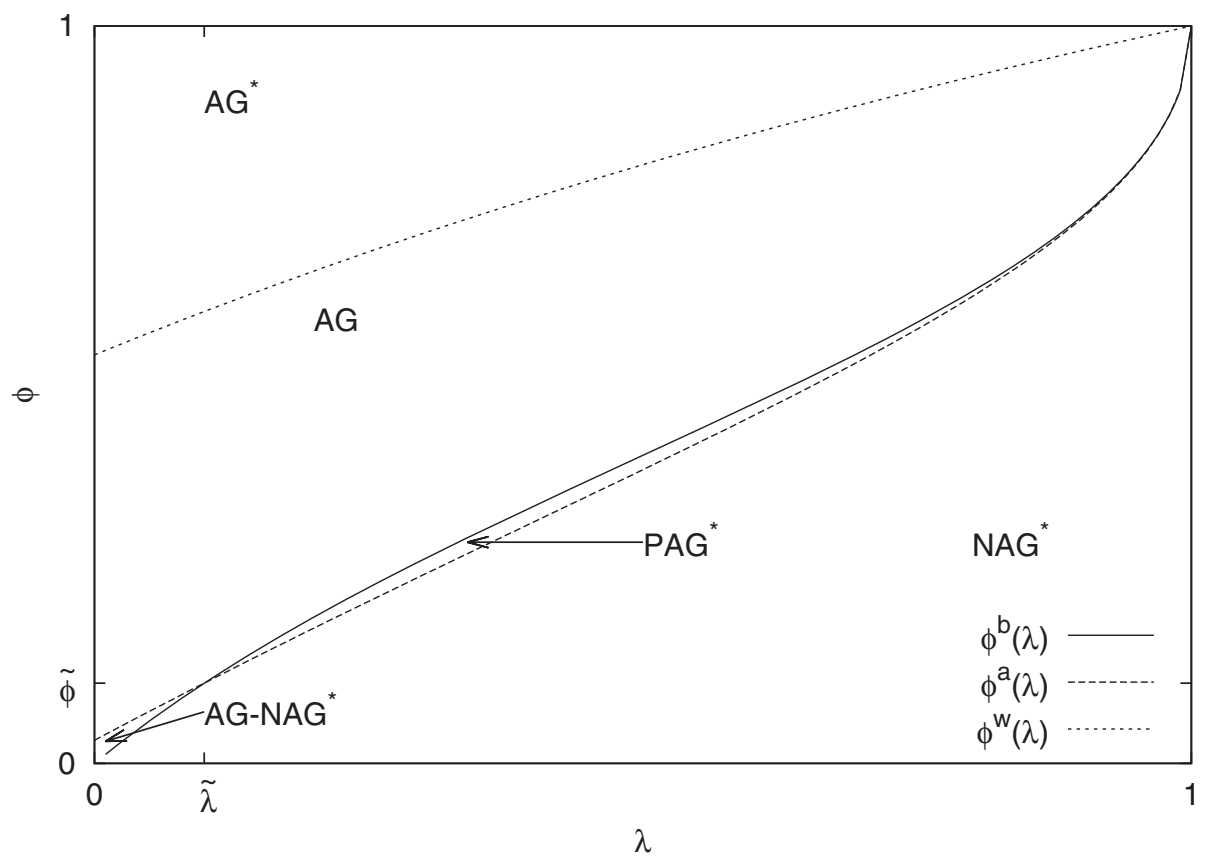

Note: Total welfare maximizers are marked with $\mathrm{a}^{*}$. They are above the line $\phi^{w}(\lambda)=\left(\rho^{w}(\lambda)\right)^{\sigma-1}$ in case of agglomerated economies and below it for nonagglomerated economies. The values of the economy parameters are the same as in Figure 3.

FIGURE 5: Maximum Welfare Equilibria.

AG is a welfare maximum provided that trade is sufficiently open. In this case, the profits generated in the agglomerated region are high enough to offset the losses due to a high-price index in the traditional region. High $\rho s$ and low $\lambda s$ are in fact, respectively, lowering the price index difference between AG and NAG and increasing the gains in terms of capital rents due to agglomeration. Notice that since $\rho^{w}(\lambda)$ is an increasing function of $\lambda$, the minimal level of freeness of trade sufficient to make AG the welfare maximum is increasing with the strength of the interregional spillover. Conversely, when $\rho<\rho^{w}(\lambda)$ the welfare maximum is given by NAG. Given these differences in welfare levels, when does the geographical economic equilibrium arising from rents maximizing behavior lead to a total welfare maximum?

Figure 5 tries to answer this question putting together the results from Propositions 2 and 3. Starred labels denote those geographical equilibria that are welfare maximizers. In the upper left area, above $\rho^{w}(\lambda)$, agglomerated economies are both a geographical equilibrium and the welfare maximum. In the lower right area, below $\rho^{a}(\lambda)$, the same is true for nonagglomerated economies. For any value of the interregional spillover there also exists an intermediate range of trade costs where NAG is the welfare optimum but the geographical equilibria are AG or PAG. There seems to be no continuous path that links the upper-right area $\mathrm{AG}^{*}$ with the lower-left area $\mathrm{NAG}^{*}$, apart from the fully integrated economy $(\lambda=1, \rho=1)$. In other terms, it is never possible to reach full trade openness following a path where the total welfare is always maximal without at the same time attaining full technological openness. The following lemma proves that the latter is a general result. 
LEMMA 3. Provided that $\widetilde{S}>0$ and $S<\min \{\widetilde{S}, \bar{S}\}$, it holds that

$$
\rho^{a}(\lambda)<\rho^{w}(\lambda), \text { for every } \lambda \in(0,1),
$$

and $\rho^{a}(\lambda)=\rho^{w}(\lambda)$ when $\lambda=1$.

\section{Welfare Enhancing Policies}

Having derived the total welfare for all values of the "openness" parameter, the next concern is how an economy can move toward the line $\rho=1$, that is, the locus of the parameters space where the total welfare is the highest. Lemma 3 tells us that the only path that brings the economy to the highest welfare passing through welfare maxima is the one that reaches full openness when $\lambda$ and $\rho$ are both one.

In general, under which conditions it is more beneficial to embrace policies that improve the freeness of trade and when, instead, it is better to increase the interregional knowledge spillovers? To answer this question assume that the government, by taxing households income $I$, can implement a policy $g$ that increases the level of freeness of trade $\rho$ and/or the interregional knowledge spillovers $\lambda$.

When the policy does not entail a change in the geographical equilibrium its effect on the total welfare for $x \leq 1 / 2$ can be computed as ${ }^{10}$

$$
\frac{\partial W_{T}(x)}{\partial g}=\frac{\partial W_{1}(x)}{\partial g}=\frac{1}{P_{1}(x)} \frac{\partial I(x)}{\partial g}+\frac{\partial W_{1}(x)}{\partial \lambda} \frac{\partial \lambda}{\partial g}+\frac{\partial W_{1}(x)}{\partial \rho} \frac{\partial \rho}{\partial g},
$$

where $\partial I(x) / \partial g$ stands for the cost of the policy. Under the assumption that a given amount of money spent by the policy $g$ has the same impact on $\rho$ and $\lambda$, i.e., $\partial \lambda / \partial g=\partial \rho / \partial g$, evaluating whether it is more welfare enhancing to increase $\rho$ or $\lambda$ amounts to compare, at the different geographical equilibria, $\partial W_{1}(x) / \partial \lambda$ with $\partial W_{1}(x) / \partial \rho .{ }^{11}$ This leads to the following lemma:

LEMMA 4. Consider the total welfare $W_{T}(x)$ as in (22). Provided that the economy is in a AG state, it is always more beneficial to increase the freeness of trade $\rho$ rather than the interregional spillover $\lambda$. Otherwise, when the economy is in a NAG state, having defined

$$
\lambda^{+}(\rho)=\frac{\frac{N \alpha}{L}+\sqrt{\left(\frac{N \alpha}{L}\right)^{2}+4 \frac{N \alpha}{L} \frac{1+\rho}{\mu \rho^{\sigma-2}}}}{2}-1,
$$

it is more beneficial to increase the freeness of trade $\rho$ when $\lambda>\lambda^{+}(\rho)$, to increase the interregional spillover $\lambda$ when when $\lambda<\lambda^{+}(\rho)$, and indifferent when $\lambda=\lambda^{+}(\rho)$.

When the modern sector is agglomerated, fixed production costs abatement does not depend on interregional spillovers, and neither does households welfare. As a result, policies that increase $\lambda$ have no effects and it is preferable to improve the freeness of trade $\rho$. Conversely, when the modern sector is evenly spread between the two regions, Lemma 4 shows that it is more welfare improving to increase interregional knowledge spillovers when $\lambda<\lambda^{+}(\rho)$, and to increase the freeness of trade $\rho$ otherwise. Figure 6 summarizes

\footnotetext{
${ }^{10}$ Given the symmetry of the welfare function around $x=1 / 2$, one can easily compute the effect of the policy also for $x \geq 1 / 2$.

${ }^{11}$ The evaluation of the policy can be complicated by a change of the geographical equilibrium caused by a change of the policy parameters $\lambda$ and $\rho$ themselves. This occurs in the case of direct transition between NAG and AG, and in the case of PAG (see, e.g., the left panel of Figure 4). Since the parameters space where this dependence occurs is small, as can be seen in Figure 3, we skip this analysis at this stage of our work.
} 


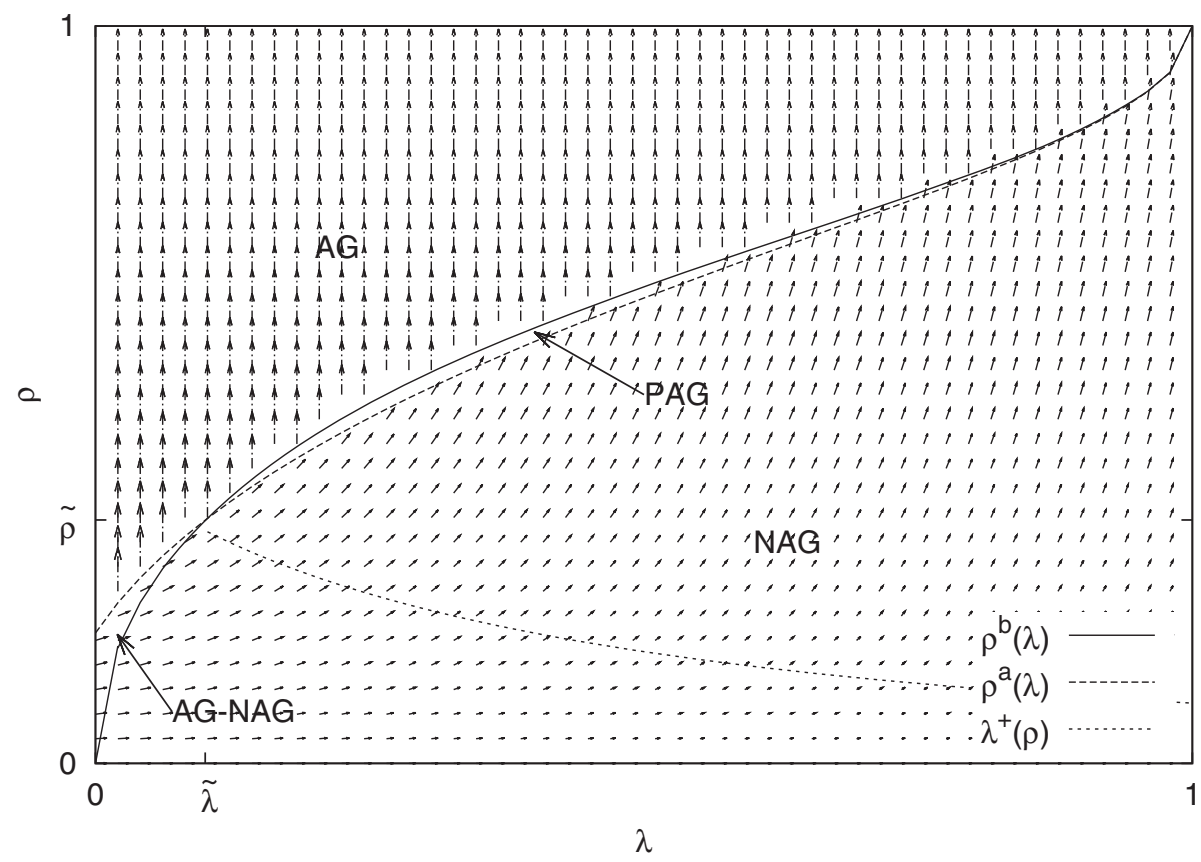

Note: $\left(\rho^{a, b}\right)^{1 / \rho-1}$. When the economy is in a AG state it is always better to increase freeness of trade. When the economy is in a NAG state, it is better to increase freeness of trade when $\rho$ is above the line $\lambda^{+}(\rho)$ and to increase inter-regional spillovers otherwise. The values of the economy parameters are the same as in Figures 3 and 5.

FIGURE 6: Gradients of the Welfare Function for NAG and AG Economies.

these results and plots the gradient of the welfare function on the plane $(\lambda, \rho)$ for our benchmark choice of the economy parameters.

\section{EXOGENOUS REGIONAL DIFFERENCES}

This last section before the conclusion explores the effects of regional exogenous differences on geographical equilibria found in Sections 3 and 4. Two forms of exogenous differences are considered. The two regions may differ in the number of households or in internal $R \& D$ costs. In the first case, we measure the difference using a parameter $\delta$ and assume that location 1 has $L(1+\delta)$ households whereas location 2 has $L(1-\delta)$ households. Without loss of generality we impose $\delta \in(0,1)$. Similarly, fixed cost differences are measured by $\epsilon \in(-1,1)$ so that fixed costs are proportional to $\alpha(1+\epsilon)$ in location 1 and $\alpha(1-\epsilon)$ in location 2 . The sign of $\epsilon$ is not restricted so that both locations can enjoy the lowest total fixed costs.

Repeating the market equilibrium analysis of Section 2 the expression for the capital rent in the two locations becomes

$$
\left\{\begin{array}{l}
r_{1}(x)=I(x) \frac{\mu L}{N \sigma}\left(\frac{1+\delta}{x+(1-x) \phi}+\frac{(1-\delta) \phi}{x \phi+(1-x)}\right)-\frac{\alpha(1+\epsilon)}{x+\lambda(1-x)} \\
r_{2}(x)=I(x) \frac{\mu L}{N \sigma}\left(\frac{1-\delta}{x \phi+(1-x)}+\frac{(1+\delta) \phi}{x+(1-x) \phi}\right)-\frac{\alpha(1-\epsilon)}{1-x+\lambda x},
\end{array}\right.
$$


where

$$
I(x)=1+\frac{\mu}{\sigma-\mu}\left(1-\frac{S}{2 \bar{S}}\left(\frac{x(1+\epsilon)}{x+\lambda(1-x)}+\frac{(1-x)(1-\epsilon)}{1-x+\lambda x}\right)\right) .
$$

As a result

$$
\Delta(x)=2 \alpha\left(I(x) \frac{\bar{S}}{S}\left(\frac{x_{\delta}-x}{x-x^{2}+\frac{\phi}{(1-\phi)^{2}}}\right)-\frac{x_{\epsilon}-x}{\left(x-x^{2}\right)(1-\lambda)+\frac{\lambda}{1-\lambda}}\right),
$$

where we have defined

$$
x_{\delta}=\frac{1}{2}+\frac{\delta}{2} \frac{1+\phi}{1-\phi}, \quad \text { and } \quad x_{\epsilon}=\frac{1}{2}+\frac{\epsilon}{2} \frac{1+\lambda}{1-\lambda} .
$$

When $\delta=0$ and $\epsilon=0$ the symmetric case of (12) and (13) is recovered. In order to keep the model tractable, we focus on the two extreme cases where the interregional spillover $\lambda$ is either 0 or 1 .

\section{Full Interregional Spillover}

When $\lambda=1$, technological spillovers operate globally and all firms "share" labor-fixed costs. Agglomeration forces are weak and, in the symmetric case, the outcome is NAG for all initial conditions. Indeed, since technological externalities are global and labor is not mobile, households firms equidistribute between the two locations. The outcome in the asymmetric case is similar in that a unique geographical equilibrium $x$ exists and attracts all trajectories irrespectively of the initial condition. In this case, however, $x$ does not need to be equal to $1 / 2$, corresponding to NAG, but it is higher or lower depending on the regional difference parameters $\epsilon$ and $\delta$ and on the freeness of trade $\phi$, as characterized by the following propositions:

PROPOSITION 4. If $\lambda=1$ there exist two values $\phi^{+}$and $\phi^{-}$of the trade openness parameter such that the geographical equilibrium is PAG if $\epsilon<0$ and $\phi<\phi^{-}$or if $\epsilon>0$ and $\phi<\phi^{+}$. The PAG equilibrium belongs to the interval $\left(x_{\delta}, 1\right)$ in the former case and to $\left(0, x_{\delta}\right)$ in the latter. Otherwise the equilibrium is $A G$, and $x=1$ when $\epsilon<0$ whereas $x=0$ when $\epsilon>0$.

When $\epsilon=0$, that is the two locations are equal in terms of fixed costs, the technological spillovers, acting globally, do not lead to agglomeration. However, because of its largest size, region 1 benefits from an home market effect and more firms locate there rather than in region 2 . Whether the home market effect leads to an interior or a border equilibrium depends on the freeness of trade. If $\phi$ is large enough the equilibrium is at 1 , otherwise it is interior. The same pattern occurs when $\epsilon<0$, with the addition that the home market effect is reinforced by having lower fixed costs in region 1. Comparatively, it will be observed that a larger fraction of firms settle in region 1 . It can also be checked that the threshold $\phi_{-}$is decreasing with $\epsilon$. The effect of asymmetries might appear more complicated when $\epsilon>0$ as firms located in region 1 face a trade-off between higher local demand, due to $\delta>0$, and higher fixed costs, due to $\epsilon>0$. However, according to Proposition 4, the overall picture turns out to be similar, with the cost saving effect playing a leading role over the home market effect. In fact, as the freeness of trade increases, it is the region where costs are lower that gains firms till the point where all firms are located in the border configuration $x=0$.

\section{No Interregional Spillover}

When $\lambda=0$, the technological spillover operates only locally. In the symmetric case, the strong agglomeration forces due to localized spillover induce an AG equilibrium with 
high freeness of trade and, possibly, a NAG equilibrium with low freeness of trade. Again regional differences shift the position of the interior equilibria but not the overall picture as shown by the following proposition:

PROPOSITION 5. Let $\lambda=0$. If

$$
\epsilon \notin\left[\frac{(\bar{S}-S) \sigma+\delta(\sigma \bar{S}-\mu S)}{(\sigma-\mu) S}, \frac{(S-\bar{S}) \sigma+\delta(\sigma \bar{S}-\mu S)}{(\sigma-\mu) S}\right]
$$

only AG occurs. Otherwise, there exists a threshold value $\hat{\phi}$ such that if $\phi<\hat{\phi} A G$ and PAG coexist. When $\phi=\hat{\phi}$ the fixed point associated to the unique interior equilibrium becomes unstable through a supercritical pitchfork bifurcation. If $\phi>\hat{\phi}$ only AG occurs.

Technological externalities are always strong enough to make agglomerated economy a geographical equilibrium. The basin of attraction of the two agglomerated equilibria and whether more equilibria exist depend however on regional differences. For large regional differences no other equilibria exist. For small regional differences, when $\phi$ is small enough there exist three geographical equilibria, the two border and one interior. Conversely, when $\phi$ is larger so that the two regions are sufficiently trade-integrated, agglomeration is the unique long-run outcome. In the extreme case when $\phi=1$, agglomeration either in 0 or in 1 is always the unique outcome and the basins of attraction of the two border equilibria are easily determined: firms agglomerate in region 1 when the initial condition is $x_{0}<$ $(1+\epsilon) / 2$ and in 2 when $x_{0}>(1+\epsilon) / 2$. In fact, as trade costs are zero and the economy is fully integrated the region with the larger labor force has no exogenous advantages anymore and basins of attraction are determined by relative fixed costs.

As with symmetric locations, also in presence of asymmetries agglomeration in either locations is always an equilibrium and when the transition between agglomeration and nonagglomeration occurs it does so via an abrupt change. This transition is, however, not the rule. Rather, it follows from the absence of interregional spillovers.

\section{CONCLUSION}

We have set-up a NEG model with capital mobility, workers intersectoral mobility and interregional immobility, and where agglomeration is due to labor enhancing technological externalities. These externalities are created by the cost saving effect of localized knowledge spillovers. We have been able to compute all geographical equilibria and basins of attraction, fully characterize their dependence on the trade-off between technological and pecuniary externalities, as regulated by transportation costs and interregional spillovers, and discuss their implications for total welfare. To retain tractability, we have considered primarily the case of symmetric regions. In the last section, we have shown that our results apply also to a priori asymmetric regions, in the extreme cases of fully local and fully global technological externalities.

Our analysis extends previous findings by Baldwin and Forslid (2000) about the stabilizing nature of knowledge spillovers: the higher the spillover the larger the interval of transportation costs that lead to firms equidistribution. Moreover, our analysis shows that if spillovers are high enough, there exists a smooth equilibrium transition between agglomeration and equidistribution, with a partly agglomerated economy for intermediate values of transportation costs. In this case, the opening of interregional trade does not entail an abrupt reallocation of economic activities neither the hysteresis effect, typical of NEG model, which locks the economy in a core-periphery equilibrium also if higher trade costs are reintroduced. 
The welfare analysis reveals that for a relatively large part of trade and technological openness parameters, even if the agglomerated outcome represents the geographic equilibrium, it generates less welfare in the periphery region than in the core. Since, in any case, the level of welfare of the periphery in the AG equilibrium increases with trade openness, for large enough level of the technological openness this solution represents the welfare optimum for both regions. However, the existence of a large "welfare gap" makes the implementation of policies based on progressive opening of the economy difficult to implement.

Conversely, the increase of the technological openness always improves the welfare level of both locations. When the level of knowledge sharing is low, its increase represents, from the point of view of the social planner, the best policy. Beside the positive effect on welfare levels, the increase of technological openness has also another advantage: for an economy with strong knowledge/technological integration, the "welfare gap" between agglomerated and nonagglomerated distribution is smaller and shallower and, consequently, policy geared toward markets integration are easier and less costly to implement.

In practice, an increase in technological openness can be obtained by improving global means of information sharing, developing joint education programs, unifying norms and requirements affecting economic activities, and relaxing institutional constraints. All these policies have the effect of improving global efficiency by avoiding replicated efforts and by abating knowledge barriers adding to transaction costs. Concluding, whereas freeness of trade leads, per se, to sudden agglomeration, knowledge-based linkages favor a smoother transition between different levels of firms concentration and ultimately lead to a less uneven distribution of welfare.

Finally, we want to express two cautionary considerations. First, we are aware that, despite the interest localized knowledge spillovers have attracted, the precise scale and scope of their action is still an open question (Rallet and Torre, 1999), as much as whether they uniquely act as positive externalities or, in the long run, they turn out to be negative externalities due to lock-in effects (Boschma, 2005). For these reasons our formulation has all the limits of a toy-model. Second, while analytical tractability allowed us to derive precise results in a general equilibrium framework, slightly different specifications of households preferences or market structure have the potential to change them, as shown by Pflüger and Südekum (2011) for the original NEG model.

\section{REFERENCES}

Audretsch, David B. and Maryann P. Feldman. 2004. "Knowledge Spillovers and the Geography of Innovation," in J. Henderson and J.-F. Thisse (eds.), Handbook of Regional and Urban Economics. Amsterdam North-Holland, pp. 2713-2739 (Handbooks in Economics Series).

Baldwin, Richard and Rikard Forslid. 2000. "The Core-Periphery Model and Endogenous Growth: Stabilizing and Destabilizing Integration," Economica, 67, 307-324.

Baldwin, Richard, Rikard Forslid, Philippe Martin, Gianmarco Ottaviano, and Frederic Robert-Nicoud. 2003. Economic Geography and Public Policy. Princeton: Princeton University Press.

Baldwin, Richard, Philippe Martin, and Gianmarco Ottaviano. 2001. "Global Income Divergence, Trade and Industrialization: The Geography of Growth Take Off,” Journal of Economic Growth, 6, 5-37.

Boschma, Ron A. 2005. "Proximity and Innovation: A Critical Assessment," Regional Studies, 39, 61-74.

Bottazzi, Giulio, Giovanni Dosi, Giorgio Fagiolo, and Angelo Secchi. 2008. "Sectoral and Geographical Specificities in the Spatial Structure of Economic Activities," Structural Change and Economic Dynamics, 19, 189-2002.

Bottazzi, Giulio and Ugo Gragnolati. 2012. "Cities and Clusters: Economy-Wide and Sector-Specific Effects in Corporate Location,” Regional Studies, DOI: 10.1080/00343404.2012.739281.

Brenner, Thomas. 2006. "Identification of Local Industrial Clusters in Germany," Regional Studies, 40, 991-1004.

Breschi, Stefano and Francesco Lissoni. 2001a. "Knowledge Spillovers and Local Innovation Systems: A Critical Survey," Industrial and Corporate Change, 10(4), 975-1005.

- 2001b. "Localised Knowledge Spillovers vs. Innovative Milieux: Knowledge 'Tacitness' Reconsidered," Papers in Regional Science, 80, 255-273. 
Davis, Colin R. 2009. "Interregional Knowledge Spillovers and Occupational Choice in a Model of Free Trade and Endogenous Growth," Journal of Regional Science, 49(5), 855-876.

Devereux, Michael, Rachel Griffith, and Helen Simpson. 2004. "The Geographic Distribution of Production Activity in Britain," Regional Science and Urban Economics, 34, 533-564.

Dixit, Avanish K. and Joseph E. Stiglitz. 1977. "Monopolistic Competition and Optimun Product Diveristy," American Economic Review, 67(3), 297-308.

Dosi, Giovanni, Keith Pavitt, and Luc Soete. 1990. The Economics of Technical Change and International Trade. New York: New York University Press.

Dupont, Vincent and Philippe Martin. 2006. "Subsidies to Poor Regions and Inequalities: Some Unpleasant Arithmetic,” Journal of Economic Geography, 6, 223-240.

Ellison, Glenn and Edward L. Glaeser. 1997. "Geographical Concentration in U.S. Manufacturing Industries: A Dartboard Approach,” Journal of Political Economy, 105, 889-927.

Forslid, Rikard and Gianmarco Ottaviano. 2003. "An Analytically Solvable Core-Periphery Model," Journal of Economic Geography, 3, 229-240.

Grossman, Gene M. and Elhanan Helpman. 1991. Innovation and Growth in the World Economy. MIT Press: Cambridge, MA.

Krugman, Paul 1991a. Geography and Trade. UK: The MIT Press.

- 1991b. "Increasing Returns and Economic Geography," The Journal of Political Economy, 99, 483-499.

Malmberg, Anders and Peter Maskell. 2006. "Localized Learning Revisited," Growth and Change, 37(1), 1-18.

Marshall, Alfred 1920. Principles of Economics. London: Macmillan and Co.

Martin, Philippe 1999. "Public Policies, Regional Inequalities and Growth," Journal of Public Economics, 73, 85-105.

Martin, Philippe and Gianmarco Ottaviano. 1999. "Growing Locations: Industry Locations in a Model of Endogenous Growth," European Economic Review, 43, 281-302.

Martin, Philippe and Carol Ann Rogers. 1995. "Industrial Location and Public Policy," Journal of International Economics, 39, 335-351.

Maurel, Francoise and Beatrice Sedillot. 1999. "A Measure of the Geographic Concentration in French Manufacturing Industries," Regional Science and Urban Economics, 29, 575-604.

Pflüger, Michael and Jens Südekum. 2011. "On Pitchforks and Tomahawks,” Journal of Regional Science, 51(2), 292-298.

Rallet, Alain and André Torre. 1999. "Is Geographical Proximity Necessary in the Innovation Networks in the Global Economy?” GeoJournal, 49, 373-380.

\section{APPENDIX: PROOFS}

\section{Proof of Lemma 1}

The proof that capital rents are as in (12) is in the text. In order to show that under condition (14) both local labor markets clear, notice that under (5) total fixed costs in each location are nondecreasing. As a result, the maximum amount of labor used by the modern sector in location $l=1,2$ is achieved when all firms are located in $l$. Consequently, condition (14) is found by imposing that the demand for labor of the modern sector, when it is totally aggregated in one region, is lower than $L$.

\section{Proof of Lemma 2}

The per capita rent $R(x)$ in (13) does not depend on $\phi$ but does depend on $\lambda$. The given value of $\bar{S}$ has been found by equating to zero the minimum values of per-capita rents, obtained when $\lambda=0$ and firms are not agglomerated, i.e., $x \in(0,1)$.

\section{Proof of Proposition 1}

Using the expressions in (12), after some simplifications, one finds

$$
\Delta(x)=\frac{(1-2 x)\left((2 a+4 b)\left(x^{2}-x\right)+b\right)}{2(\sigma-\mu)(x+\phi(1-x))(x \phi+1-x)(x+\lambda(1-x))(x \lambda+1-x)},
$$


where

$$
\begin{aligned}
& a=(1-\phi)^{2}\left(\alpha N \sigma(1-\lambda)-\mu L(1+\lambda)^{2}+2 \alpha N \mu \lambda\right)+4 \alpha N \phi(1-\lambda)(\sigma-\mu), \\
& b=\mu \lambda(1-\phi)^{2}(2 L-\alpha N)-2 \alpha N \phi(1-\lambda)(\sigma-\mu) .
\end{aligned}
$$

We can restrict our analysis to the signs and derivatives of $N(x)$, the numerator of $\Delta(x)$. Indeed, according to our hypothesis $\sigma>\mu$ and $\phi, \lambda \in(0,1]$, so that the denominator of $\Delta(x)$ is always positive implying that both the signs and zeros of the rent difference are equal to the signs and zeros of its numerator. Moreover, the sign of the derivative of the rent difference evaluated at its zeros is equal to the sign of the derivative of its numerator evaluated at the same points.

First notice that $N(x)$ is a cubic function and symmetric around $x=1 / 2$. It follows that the dynamics in (16) has at most five different fixed points: the two extreme values 0 and 1 and the three zeros of $N(x)$. Its parametrization in terms of $a$ and $b$ has been chosen so that

$$
\begin{gathered}
a=N^{\prime}(1 / 2)=\Delta^{\prime}(1 / 2), \\
b=N(0)=\Delta(0) .
\end{gathered}
$$

As a result, when $a$ and $b$ are both positive, the derivative of the rent difference computed at $x=1 / 2$ is positive, the rent difference at $x=0$ is positive and, by symmetry, negative at $x=1$. Given that $N(x)$ is a cubic polynomial, it must be zero in two other points in the interval $[0,1]$, which we name $x^{+}$and $x^{-}$and are symmetrically located around $1 / 2$. Moreover, the marginal rent difference at both $x^{+}$and $x^{-}$must be negative, so that these points are indeed geographical equilibria corresponding to PAG. It turns out that this is the only sign combination for which the economy is in a PAG status, as can be easily checked by repeating the same reasoning for all the other sign combinations of $a$ and $b$. The signs of $N(x)$, and thus also of $d x / d t$, shows that the system converges to $x^{-}$for initial conditions $x_{0} \in(0,1 / 2)$ and to $x^{+}$for $x_{0} \in(1 / 2,1)$. All other results follow along the same lines, see also Figure 2.

\section{Proof of Proposition 2}

As with the previous proof we characterize the signs of $\Delta(x)$ and $\Delta^{\prime}(x)$ by looking at the signs of its numerator $N(x)$ and its derivative $N^{\prime}(x)$. The equations $\Delta^{\prime}(1 / 2)=0$ and $\Delta(0)=0$ can be solved in terms of $\phi$ giving

$$
\begin{aligned}
& \phi_{ \pm}^{a}(\lambda)=\left(1+\Gamma(\lambda) \pm \sqrt{\Gamma^{2}(\lambda)+2 \Gamma(\lambda)}\right)^{\frac{1}{\sigma-1}}, \\
& \phi_{ \pm}^{b}(\lambda)=\left(1+\Theta(\lambda) \pm \sqrt{\Theta^{2}(\lambda)+2 \Theta(\lambda)}\right)^{\frac{1}{\sigma-1}},
\end{aligned}
$$

with

$$
\begin{aligned}
\Gamma(\lambda) & =\frac{\alpha N(1-\lambda)(\sigma-\mu)}{\mu \lambda(2 L-\alpha N)+\frac{1-\lambda}{2}(\mu L(1-\lambda)-N \alpha \sigma)}, \\
\Theta(\lambda) & =\frac{\alpha N(1-\lambda)(\sigma-\mu)}{\mu \lambda(2 L-\alpha N)} .
\end{aligned}
$$

Provided that $S=N / L<\bar{S}$ one can show that both $\Gamma(\lambda)$ and $\Theta(\lambda)$ are positive for any value of $\lambda$. This, in turn, implies that both $\phi_{+}^{a}$ and $\phi_{+}^{b}$ are larger than 1 for every $\lambda$ in $[0,1]$, whereas $\phi_{-}^{a}$ and $\phi_{-}^{b}$ are two functions from $[0,1]$ to $[0,1]$ and correspond to $\phi^{a}(\lambda)$ and $\phi^{b}(\lambda)$ given in (17) and (18). Taking the limits to 1 and 0 it holds $\phi^{b}(1)=\phi^{a}(1)=1$ and $\phi^{b}(0)=0$. 
Furthermore, substituting $\tilde{\lambda}$ in $\Theta(\lambda)$ and $\Gamma(\lambda)$ it is immediate to check that $\phi^{a}(\tilde{\lambda})=\phi^{b}(\tilde{\lambda})$ and that $\phi^{b}(\tilde{\lambda}) \gtreqless \phi^{a}(\tilde{\lambda})$ when $\lambda \gtreqless \tilde{\lambda}$. Notice at last that since $S<\bar{S}$, it always holds that $\tilde{\lambda} \in(0,1)$.

\section{Proof of Proposition 3}

The proof relies on the properties of income $I(x)$ and of price indexes $P_{1,2}(x)$. For the income function defined in (13) one can easily show that $I(x)=I(1-x), I^{\prime}(1 / 2)=$ $0, I^{\prime}(x) \gtreqless 0$ when $x \gtreqless 1 / 2$, and $I^{\prime \prime}(x)>0$. For the price index functions in (21) it holds $P_{1}(x)=P_{2}(1-x), P_{1}^{\prime}(x)=-P_{2}^{\prime}(x)<0$, and $P_{1}^{\prime \prime}(x)=P_{2}^{\prime \prime}(x)>0$. Using these properties we can rewrite the expression of the max-min welfare as

$$
W_{T}(x)= \begin{cases}W_{1}(x) & x \leq \frac{1}{2} \\ W_{2}(x) & x \geq \frac{1}{2}\end{cases}
$$

Due to the symmetry of the economy, we can restrict our attention to the maxima of $W_{2}(x)$ in the interval $[1 / 2,1]$. Given the behavior of $I(x)$ and $P_{2}(x)$, it holds both that $W_{2}^{\prime}(1 / 2)<0$ and that there exists at most one value of $x \in[1 / 2,1]$ where $W_{2}^{\prime}(x)=0$. As a result, the global maxima of the continuous and differentiable function $W_{2}(x)$ in the interval $[1 / 2,1]$ are on its border, that is, either $x=1 / 2$ or $x=1$. In order to determine which of the two prevails, we compare their welfare level and find

$$
\frac{W_{2}(1)}{W_{2}(1 / 2)} \gtreqless 1 \Leftrightarrow \rho \gtreqless \rho^{w}(\lambda)=\left(2\left(\frac{L-\frac{N \alpha}{2}}{L-\frac{N \alpha}{1+\lambda}}\right)^{\frac{\sigma-1}{\mu}}-1\right)^{-\frac{1}{\sigma-1}} .
$$

\section{Proof of Lemma 3}

By evaluating $\rho^{a}(\lambda)$ and $\rho^{w}(\lambda)$ in $\lambda=1$, one immediately obtains that they are both equal to one. The rest of the statement has been proved numerically. We have defined a grid of 500 values of $\sigma$ in $(1,100], 100$ values of $\mu$ in $(0, \sigma /(2 \sigma-1))$, which ensures that $\widetilde{S}>0,100$ values of $S$ in $(0, \min \{\widetilde{S}, \bar{S}\})$, and 500 values of $\lambda$ in $[0,1)$. We have checked that for every set of values it holds that

$$
\begin{array}{ll}
\frac{d \rho^{w}(\lambda)}{d \lambda}<\frac{d \rho^{a}(\lambda)}{d \lambda} & \text { when } \lambda<\tilde{\lambda}, \\
\frac{d \rho^{w}(\lambda)}{d \lambda}<\frac{d \rho^{b}(\lambda)}{d \lambda} & \text { when } \lambda \geq \tilde{\lambda} .
\end{array}
$$

The two inequalities, together with the fact that all these curves are equal to one when $\lambda=1$, monotonic, and $\rho^{a}(\lambda) \gtreqless \rho^{b}(\lambda)$ when $\lambda \gtreqless \tilde{\lambda}$, see Proposition 2 , prove the result.

\section{Proof of Lemma 4}

The gradient of the total welfare at the two geographical equilibria corresponding to NAG and AG is:

$$
\frac{\partial W_{T}(x)}{\partial \lambda}= \begin{cases}\left(\frac{N \alpha \sigma}{(\sigma-\mu) L(1+\lambda)^{2}}\right)\left(\frac{\sigma-1}{\beta \sigma}\left(\frac{N}{2}\right)^{\frac{1}{\sigma-1}}\right)^{\mu}(1+\rho)^{\frac{\mu}{\sigma-1}}, & x=0.5 \\ 0 & x=0,1\end{cases}
$$




$$
\frac{\partial W_{T}(x)}{\partial \rho}= \begin{cases}\left(\frac{\sigma \mu}{\sigma-\mu}-\frac{N \alpha \sigma \mu}{(\sigma-\mu) L(1+\lambda)}\right)\left(\frac{\sigma-1}{\beta \sigma}\left(\frac{N}{2}\right)^{\frac{1}{\sigma-1}}\right)^{\mu}(1+\rho)^{\frac{\mu}{\sigma-1}-1} \rho^{\sigma-2}, & x=0.5, \\ \left(\frac{\sigma \mu}{\sigma-\mu}-\frac{N \alpha \sigma \mu}{(\sigma-\mu) 2 L}\right)\left(\frac{\sigma-1}{\beta \sigma}(N)^{\frac{1}{\sigma-1}}\right)^{\mu} \rho^{\mu-1}, & x=0,1 .\end{cases}
$$

Since for AG economies $\partial W_{T} / \partial \lambda$ is zero the best policy is always to increase the freeness of trade. The result for NAG economies follows from the comparison of the two components of its gradient.

\section{Proof of Proposition 4}

The rent difference $\Delta(x)$ in (27) can be written as the ratio of two polynomials. If $\lambda=1$, the denominator is always positive, so that the study of the second-order polynomial in the numerator $N(x)$ is sufficient.

When $\epsilon<0, N(0)>0$, and $N^{\prime}(x)<0$. The polynomial possesses a single root in $(0,1)$ provided that $N(1)<0$. This root is a globally attracting internal fixed point. Conversely, if $N(1) \geq 0$ the system agglomerates in $x=1$. Solving $N(1)=0$ for $\phi$ leads to the identification of $\phi^{-}$. The location of the PAG equilibrium in the interval $\left(x_{\delta}, 1\right)$ follows from noticing that $N\left(x_{\delta}\right)>0$.

When $\epsilon<0, N(1)<0$, and $N^{\prime}(x)>0$. A similar reasoning leads to the identification of $\phi^{+}$and to the statement.

\section{Proof of Proposition 5}

As long as $\phi>0, \Delta(x)<0$ in a right neighborhood of 0 and $\Delta(x)>0$ in a left neighborhood of 1 so that the two border equilibria always exist. Moreover, since the denominator of $\Delta(x)$ is always positive and its numerator, $N(x)$, is a third-order polynomial, there exists at most one additional interior geographical equilibrium.

Consider the extreme case $\phi=0$. The capital rent difference reads

$$
\Delta(x)=\frac{2 \alpha \sigma(\bar{S}-S)}{(\sigma-\mu) S}\left(\frac{x_{0}-x}{x-x^{2}}\right), \quad \text { where } \quad x_{0}=\frac{1}{2}+\frac{\delta}{2}+\frac{(\sigma-\mu) S(\delta-\epsilon)}{2 \sigma(\bar{S}-S)} .
$$

When $x_{0} \geq 1$, the only globally stable fixed point is $x=1$. Analogously, when $x_{0} \leq 0$, the only globally stable fixed point is $x=1$. When $x \in(0,1)$, which occurs when regional asymmetries are not too big, $x_{0}$ is the unique interior geographical equilibrium. Since the numerator $N(x)$ is a smooth function of $\phi$ we can conclude that in the latter case $\Delta(x)$ has a zero close to $x_{0}$ for $\phi$ close enough to 0 . Moreover, its first-order differential keeps the same sign as $N^{\prime}\left(x_{0}\right)<0$. Then, since $N(x)$ is negative for $x=0$ and positive for $x=1$, there will be two other roots in the interval $(0,1)$. In this case, the two border equilibria and the interior equilibrium coexist. Moreover, as $\phi$ increases the root of $N(x)$ in $[0,1]$ reduces from three to one and there exists a $\hat{\phi}$ where the supercritical bifurcation occurs, so that irrespectively of the value of $x_{0}$ when $\phi$ is close to one the unique root becomes $x_{\epsilon}$ and only AG occur.

The bound on the value of $\epsilon$ given in the proposition has been found by imposing $x_{0} \notin[0,1]$. 